\title{
Os efEITOS DA APRENDIZAgEM PSICOMOTORA NO CONTROLE DAS ATIVIDADES DE LOCOMOÇÃO SOBRE OBSTÁCULOS EM CRIANÇAS COM DEFICIÊNCIA DA VISÃO
}

THE EFFECTS OF PSYCHOMOTOR LEARNING ON THE CONTROL OF LOCOMOTION DURING OBSTACLE AVOIDANCE IN CHILDREN WITH LOW VISION

\author{
Luiz Cezar dos SANTOS \\ Janine Eliza deOliveira Silva PASSOS² \\ Alexandre Luiz Gonçalves deREZENDE ${ }^{3}$
}

RESU M O : considerand o quea visão é um dos sistemas sensoriais mais importantes na locomoção pois fornece informação tanto do ambientequanto da postura e dos movimentos corporais, este estudo anal isou os efeitos de um programa de treinamento perceptivo-motor no controle das atividades de locomoção em seis crianças com deficiências da visão. Para tanto, realizou-sea aval iação da locomoção durantea transposição deobstáculos com al turas diferentes, utilizando-se indicadores qualitativos: postura/ equilíbrio / direção / velocidade/ contato visual com o obstáculo / erro na tarefa, e indicadores cinemáticos, obtidos por meio de filmagem (SVHS) através do sistema Peak M otus (Versão 7.0 - Vicon Peak) para medir: a distância do pé de apoio em relação ao obstáculo / a elevação máxima do joel ho / a altura do pé em relação à altura do obstáculo. Os indicadores foram novamente avaliados após a realização do programa detreinamento, fornecendo evidências de sua contribuição positiva tanto na estratégia de aproximação quanto na estratégia deultrapassagem dos obstáculos. Durante a aproximação, a velocidade da locomoção tornou-semais constantee as passadas foram real izadas de forma mais retilínea. Durante a ultrapassagem, o número de toques no obstáculo diminuiu. Os resultados auxiliam na reflexão sobrea importância da aprendizagem psicomotora nos programas educacionais, particularmente na Educação Física Especial, como uma forma de contribuir para uma mel hor qualidade de vida das crianças com baixa visão.

PA LA V RAS-CH A VES: atividades delocomoção; deficiências da visão; aprendizagem psicomotora, educação física; educação especial.

\begin{abstract}
A BSTRACT: since vision is one of the most important sensory systems called upon for locomotion because it provides information not only about the environment, but also about posture and movement of corporal segments, this study analyzed the effects of a psychomotor training program on the control of locomotion with six children with low vision. Thus, an assessment of locomotion during obstacle avoidance with different obstacle heights was performed based on the qualitative parameters: posture/ balance/ direction / vel ocity / visual contact with the obstacle / error on the task, and on kinematics parameters collected via motion analysis system (Peak M otus - 7.0 - Vicon Peak) to assess: the distance of the base foot in relation to obstacle position / the maximum elevation of the knee / the height of the foot in relation to obstacle height. The parameters were again analyzed after thetraining program, showing evidence of the positiveeffect of both the strategy of approximation and the strategy of going over theobstacle. On approximation, the walking velocity becamemorestableand the subjects walked straighter. During obstaclecrossing, there was less contact between the foot and the obstacle. These results will hel p to understand the importance of psychomotor learning in educational programs, especially on adapted physical education, so as to contributeto better quality of life for children with low vision.
\end{abstract}

KEYWO RD S: locomotion activities; visual impairment; psychomotor learning; physical education; special education.

${ }^{1}$ Ph.D.; Laboratório de Controle Motor; Faculdade de Educação Física; Universidade de Brasília Icsantos@unb.br

${ }^{2}$ Especial ista em Aspectos Teórico-M etodológicos da Pesquisa em Educação Física, Centro deEnsino Especial de Deficientes Visuais - CEEDV. Secretaria deEstado deEducação do Governo do Distrito Federal.

${ }^{3}$ Doutor em Ciências da Saúde - UnB, Laboratório deEducação Física Escolar, Faculdade de Educação Física, Universidade deBrasília. Campus Universitário Darcy Ribeiro - rezende@unb.br 


\section{INTRODUÇÃo}

A deficiência visual é classificada, de acordo com o DSM IV, em dois tipos: cegueira ebaixa visão. A cegueira é definida como a perda total da visão até a ausência de projeção de luz. A baixa visão é definida como a perda severa da visão eapresenta uma acuidade visual inferior a 20/ 60 ou campo visual inferior a 10 graus do seu ponto de fixação, deforma que não podeser corrigida por meio do uso de ócul os convencionais, por tratamento clínico nem mesmo por intervenção cirúrgica (APA, 1996).

O portador de baixa visão, dependendo da patologia, apresenta comprometimentos relacionados à diminuição da acuidadee/ ou campo visual, à adaptação àluz e ao escuro eà percepção de cores (CA VA LCA NTE, 1995), mas, por outro lado, utiliza, ou é potencial mentecapaz deutilizar, a visão remanescente para planejamento e execução detarefas.

Dados da Organização Mundial de Saúde(OMS) revelam a existência deaproximadamente 40 milhões de pessoas deficientes visuais no mundo, edestes, $75 \%$ em regiões consideradas em desenvolvimento. O Brasil apresenta uma taxa deincidência de deficiência visual entre 1,0 a 1,5\% da população. A estimativa de ceguei ra infantil é de 1 entre 3.000 crianças e de 1 entre 500 crianças para baixa visão(MEC/ SEE, 2001, p.39). Segundo dados da Sociedade Brasileira de Visão Subnormal , 70 a $80 \%$ das crianças inicialmente diagnosticadas como cegas, na verdade possuem al guma visão útil (SBVS, 2007).

O aproveitamento do potencial visual remanescente das crianças com baixa visão raramenteé uma questão que depende exclusivamente da maturação ou que se desenvolve de forma espontânea a partir das atividades rotineiras. Sendo assim, toda criança com baixa visão deve ser submeti da a um programa especial deestimulação, que auxilieno desenvolvimento do processo de discriminação de formas, contornos, figuras esímbolos.

A lém das questões diretamente relacionadas à visão, deve-seconsiderar que existeuma interação muito próxima entre visão e desenvolvimento, deforma que a dimi nuição das capacidades visuais implica uma série de comprometimentos em outras áreas do comportamento infantil, dentreelas a área motora.

A visão possui um papel chaveno desenvolvimento motor, pois tanto coloca a criança em contato com a reali dade externa como I he fornece estímulos que auxiliam na orientação e no controle da ação corporal. Grande parte das experiências da criança na exploração do meio, na descoberta do próprio corpo, no contato com os objetos ena rel ação com os outros, tem uma participação direta da visão, que setorna um elemento chavena organização da ação motora voluntária, consciente esegura.

A diminuição severa da visão, portanto, implica uma situação oposta que coloca a criança em uma séria situação de risco: a falta de estímulos pode acarretar uma série de déficits em funções nas quais a criança, a priori, não possui 
nenhum tipo decomprometimento, destacando a importância do acompanhamento precoce das crianças que estão nessa condição (AMARAL; TABAQUIM; LAMÔNICA, 2005).

Observa-se, por exemplo, que as vezes os bebês cegos, às vezes, apresentam um atraso no desenvol vimento motor, pois I hes faltam estímulos que provoquem a necessidade ou a vontade de se movimentar; geral mente os bebês com visão normal elevam a cabeça ou engatinham para pegar al go que eles vêem e que Ihes desperta o interesse. Os bebês cegos, no entanto, se não forem adequadamente estimulados, ficam quietos e imóveis por muito tempo, apresentando um repertório pobre de vivências motoras, que posteriormente prejudica o domínio das habilidades corporais.

Deve-setambém considerar que o movimento, além de abranger atos motores, assume uma dimensão social. LeBoulch (1988) chama a atenção para o fato de que os movimentos expressivos do corpo eas suas reações tônicas assumem uma dimensão de linguagem, construindo um elo de aproximação com o outro.

Mover-se, portanto, possibilita à criança tanto exercitar a autonomia de tentar entrar em contato com os outros, como desperta o interesse do outro de seaproximar e buscar uma interação mai or. O estudo deH ueara, Souza eBatista (2006) demonstram que a interação entre as crianças com baixa visão em situações de brincadeira proporciona um ambiente favorável às múltiplas elaborações cognitivas das crianças.

Ciente disso, torna-seimportanteestudar: 1) as dificuldades das crianças com baixa visão para utilizar o potencial visual remanescente na avaliação das circunstâncias ambientais e no controle das ações motoras adaptativas, e; 2) a estimulação adequada para semaximizar a articulação do potencial visual com as informações advindas do uso dos outros sentidos, a fim de estabelecer uma adequada avaliação do ambientee uma coordenação eficiente dos movimentos.

Portanto, esteestudo busca fornecer subsídios para a melhor adequação dos programas de educação precoce edereabilitação. A escola, ao ensinar a criança a usar a visão, investe na formação de um sentimento de autoconfiança e na melhoria das habilidades de orientação e mobilidade, dando suporte para que a pessoa adote uma postura comprometida com uma ação exploratória ena medida do possível independente, o que representa, a longo prazo, uma verdadeira conquista da cidadania e da inserção social .

Sendo assim, o presente estudo tem por objetivo contribuir para a análise dos aspectos que interferem no controle visual da locomoção em crianças com baixa visão, por meio da análise dos efeitos de um programa de estimulação proprioceptiva na mel horia da coordenação dos movimentos durantea locomoção sobreobstáculos. 


\subsection{Aspectos Perceptivo-motores que interferem na locomoção sobre obstáculos}

Durante a locomoção, quando a pessoa deve fazer a ultrapassagem por cima de um obstáculo, são necessárias diversas adaptações nos movimentos. Os estudos sobre controle visual da locomoção sobre obstáculos (PATLA et al., 1991, 1997, 1998) destacam duas variáveis que devem ser moduladas: 1) o posicionamento do pé antes do obstáculo, e; 2) a elevação da perna durante a ultrapassagem. A justes nestas duas variáveis caracterizam o processo deintegração perceptivo-motor que, associado com informações precisas sobre a posição, altura etipo deobstáculo, permitem a execução de um movimento correto.

Normalmente, como as informações sobre posição e características de um obstáculo estão previamente disponíveis durante a fase de aproximação, a pessoa pode planejar com antecedência as estratégias a serem utilizadas para ultrapassá-lo. N este caso, a informação visual é utilizada através de um controle antecipado (Feed-forward). Patla (1998) concluiu que o baixo índice de sucesso durante a ultrapassagem de um obstáculo, quando a pessoa não tem acesso às informações visuais na fase deaproximação, éatribuíd a à dificuldadedeseplanejar a estratégia adequada para o posi cionamento do péantes do obstáculo.

Estudos realizados por Chou e Draganich (1998a, b) mostraram que quando o posicionamento do péémuito próximo ao obstáculo, existe uma redução na flexão do quadril, joel ho e tornozelo. Essas mudanças na configuração geométrica da perna de apoio causaram: 1) uma diminuição na el evação do pé, em relação à altura do obstáculo, e; 2) uma redução no tempo de vôo. Tais conseqüências aumentam o risco da pessoa tropeçar no obstáculo com a perna detrás. Posicionar o péde apoio muito longe do obstáculo podetambém ser prejudicial, pois, aumenta o risco do toque do pé no obstáculo durante a ultrapassagem da perna da frente. Portanto, para uma ultrapassagem segura tanto da perna da frente quanto da perna detrás énecessário um posicionamento adequado na fase de aproximação.

Os efeitos da qualidadeda informação visual durantea locomoção sobre obstáculos foram recentemente analisados por Chapman e Hollands (2006) em um estudo com idosos durantea locomoção sobreal vos marcados no solo. O estudo mostrou quea manipulação da informação visual em diferentes momentos dentro do ciclo da passada causou efeitos significativos no posicionamento dos pés ena realização da tarefa para o grupo de idosos. Quando a informação visual foi disponível somente na fase de apoio o erro na real ização da tarefa foi cerca de $23 \%$. No momento em que a informação visual esteve disponível somente na fase de balanço o erro aumentou ainda mais (42\%). Os resultados mostram que o declínio da capacidade visuo-motora, causado pel o processo de envel hecimento, altera a forma como os idosos utilizam a informação visual para planejar e executar tarefas motoras. Os autores ressaltam que os idosos necessitam da informação visual em momentos específicos do ciclo da passada para planejar e realizar os movimentos. Os resultados mostram ainda quea diminuição da capacidadevisual écompensada pela utilização contínua (durantea real ização do movimento) da informação visual . 
A elevação da perna durantea ultrapassagem também éafetada quando a pessoa não tem informações visuais em rel ação a vários aspectos, dentre eles a informação sobre a al tura do obstáculo (PATLA; RIETDJYK. 1993; PATLA et al., 1991). Diante de obstáculos al tos, a distância entre o pée o topo do obstáculo (Toe Clearance) aumenta (PA TLA; RIETDJYK, 1993), gerando, conseqüentemente, um aumento da elevação máxima da perna (MOHAGHEGHI; MORAES; PATLA , 2003). Este aumento representa uma margem de segurança, cal culada pela pessoa de forma a garantir a ultrapassagem segura do obstáculo.

Essa margem de segurança é alterada quando a qualidade da informação visual sobre o obstáculo é bloqueada artificialmente (PATLA, 1998; MOHAGHEGHI; MORAES; PATLA, 2003). Quando a visão é bloqueada duas passadas antes do obstáculo, a margem de segurança aumenta significativamente. Isto também acontece quando a visão dos membros inferiores é removida no momento da ultrapassagem do obstáculo (PATLA, 1998).

Um estudo realizado com idosos com maculopatia (PATLA et al., 1995), que, de certa forma podem ser comparados às crianças com baixa visão, mostrou al terações significativas no controle motor dos movimentos durante a ultrapassagem. Comparados com um grupo controle os idosos com maculopatia apresentaram um posicionamento do pé de apoio mais afastado do obstáculo e uma maior el evação do pédurantea ultrapassagem do obstáculo (ToeClearance). As duas alterações são explicadas pelos autores como uma estratégia utilizada pelos idosos para obter um maior tempo para realizar correções durante a ultrapassagem, garantindo assim uma ultrapassagem mais segura.

Com base nas conclusões desses estudos, pretende-se verificar se a criança com baixa visão é capaz de aprender a utilizar estímulos proprioceptivos, associados ao potencial visual remanescente, para re-elaborar eexecutar um plano de ação mais adequado na locomoção sobre obstáculos. A melhoria do controle motor será evidenciada através de uma maior constância no posicionamento do pé mais próximo em relação ao obstáculo, e, paralelamente, de uma menor variabilidade na elevação da perna durante o momento da ultrapassagem do obstáculo. Esses indicadores são usados para informar se a estimulação proposta contribui ou não para a melhoria na confiança, na autonomia ena independência da locomoção da criança.

\section{MÉTOdo}

\subsection{Participantes}

Participaram desteexperimento seis crianças com idadedecinco a oito anos, alunos do Centro de Ensino Especial de Deficientes Visuais (CEEDV) de Brasília-DF, selecionadas de acordo com a avaliação do diagnóstico de baixa visão e sem comprometimentos cognitivos ou motores. A participação das crianças foi condicionada ao consentimento dos pais ou responsáveis. O estudo foi aprovado 
pelo ComitêdeÉtica da FaculdadedeM edicina da UniversidadedeBrasília (CEPFM 029/ 2005).

A amostra foi composta por sujeitos com diferentes patologias.

Sujeito 1- portador de Glaucoma, apresenta perda acentuada da visão periférica e da nitidez da visão central (tubular), visão monocular (olho bom: esquerdo) e redução na clareza de detal hes. A presenta também visão flutuanteefaz uso deóculos.

Sujeito2- portador de Coriorretinite Macular Bilateral, apresenta comprometimento da visão central, redução na clareza de detal hes e baixa acuidade visual para longe. A presenta também escotomas centrais, alteração na visão de cores, dificuldade para ver objetos grandes e pequenos e problemas na fixação einterpretação deimagens.

Sujeito 3 - portador deA trofia do Nervo Óptico Bilateral, apresenta baixa acuidade visual para longe, comprometimento de um quadrante da visão e redução na clareza de detalhes. Possui dificuldade para integração da imagem visual eparaformação deimagens mentais. Faz uso deóculos.

Sujeito 4 - portador de Hipermetropia em ambos os olhos 7,5, apresenta comprometimento da visão de perto epossuindo baixa fixação. Possui nistagmo eacentuada baixa de acuidade visual. Real iza grande esforço para acomodar a imagem na retina e apresenta visão embaçada e flutuante. Faz uso deóculos.

Sujeito 5 - portador deCatarata Congênita, com redução na nitidez das imagens, visão embaçada, redução na clareza de detal hes evisão para cores. Faz uso de óculos. O campo visual e a noção de profundidade não estão comprometidos.

Sujeito 6 - o portador de A nirídia, Sua visão é melhor à noite ou em ambientes com baixa intensidade de luz. Possui dificuldades de acomodação, fixação, ena interpretação deimagens e cenas.

\subsection{Programa de treinamento}

O programa de treinamento foi aplicado pelos próprios professores de Educação Física do CEEDV, duas vezes por semana, durante as aulas deEducação Física, totalizando vintesessões. Segundo Souza (2004), o programa detreinamento deve ser funcional, realizado individual mente e num ambiente adequado. $\mathrm{O}$ envolvimento dos professores da escola possibilitou a criação de um ambiente educativo familiar e afetivamente seguro, além de validar ecologicamente o programa.

A sessão de treinamento tinha duração de quinze minutos, sendo realizada com supervisão direta econstante do professor. A s crianças real izaram 
as aulas descalças e recebiam feedback do professor após cada execução. As atividades enfatizaram a ultrapassagem de obstáculos variados em situações também variadas, tomando o cuidado, porém, para não utilizar os mesmos obstáculos do teste nem de reproduzir a mesma tarefa realizada no teste desta pesquisa.

O treinamento incluiu diversas atividades sendo real izadas tanto com os ol hos abertos, estimulando o uso do potencial visual remanescente, quanto com os olhos fechados, estimulando o uso compensatório das outras fontes de informações perceptivas do ambiente e do próprio corpo. As atividades foram as seguintes: subir e descer escadas; transpor pneus e objetos de alturas variadas; e elevar os joel hos de forma al ternada eem al turas diferentes.

\subsection{NSTRUMENTOS}

A análise cinemática da locomoção foi realizada através de filmagem (SVHS), na freqüência de $60 \mathrm{~Hz}$, no plano sagital, por meio de um sistema deanál ise do movimento Peak M otus (V ersão 7.0 - V icon P eak). Foram demarcados três pontos com adesivo branco: hálux direito, joel ho direito, e hálux esquerdo para posterior digitalização.

As coletas foram realizadas na sala de Educação Física do CEEDV . A tarefa constou de uma caminhada de aproximadamente 2 metros e, ao final, da ultrapassagem de um obstáculo. A aproximação do obstáculo (perna de apoio) foi real izada sempre com a perna esquerda ea ultrapassagem inicial do obstáculo foi real izada sempre com a perna direita (perna da frente). O obstáculo utilizado foi um retângulo de espuma ( $40 \mathrm{~cm}$ delargura, $10 \mathrm{~cm}$ decomprimento) com três alturas diferentes: 5 , 10 e 15 centímetros.

Foram filmadas dez tentativas, sendo três para os obstácul os de 5 e 15 $\mathrm{cm}$ e quatro para o obstáculo de $10 \mathrm{~cm}$ de altura. Duas sessões de teste foram realizadas: uma antes e uma após o programa de treinamento.

As variáveis analisadas foram: a distância do pé de apoio (esquerdo) em relação ao obstáculo (DPO), a elevação máxima do joel ho direito durante a ultrapassagem (EMJ) ea distância entreo dedo do pédireito eo topo do obstáculo (ToeClearance).

Os dados foram analisados de forma individual tendo em vista as diferentes patologias existentes na amostra eo número reduzi do de participantes. Com basenos dados cinemáticos foram cal culadas as variações existentes entre os valores obtidos no pós-teste em relação ao pré-teste. Ao final , apresentam-se os val ores da média e desvio padrão sem, no entanto, querer dessa maneira sugerir que os resultados podem ser generalizados para outros sujeitos que não os que participaram da amostra. 
Paralelo à análisequantitativa dos indicadores cinemáticos relacionados à marcha, foi utilizado um conjunto de seteindicadores qual itativos para aval iar o desempenho das crianças na real ização da tarefa-teste. Os indicadores foram: a postura, o equilíbrio, a direção e a velocidade na realização dos movimentos; o contato visual com o obstácul o e o erro na execução da tarefa (tocar ou derrubar o obstáculo). A descrição detal hada de cada indicador éapresentada a seguir:

1) Manter o contato visual com o obstáculo: aspecto considerado chave para reforçar o princípio da otimização do uso do potencial visual remanescente para realização da tarefa-teste (todos devem apresentar);

Os demais indicadores possuem a mesma definição erepresentam aspectos que revelam as dificuldades no controle motor geradas pela falta de informações que auxiliem na execução correta da tarefa-teste (apresentam variação de um sujeito para outro).

2) Direção da caminhada na fase de aproximação do obstáculo: caminhada lateral izada (quando o pé ultrapassa o obstáculo pela lateral demonstrando flexão inadequada do joel ho);

3) Equilíbrio durante a ultrapassagem do obstáculo: utilização dos braços para auxiliar na recuperação do equilíbrio perdido;

4) Direção da passada no momento da ultrapassagem do obstáculo: posição do pélateral izada com a direção da caminhada;

5) Velocidadena execução da tarefa-teste: aspecto relacionado à segurança eà agilidadena real ização dos movimentos (apresentam variação de um sujeito para outro);

6) Tocar ou derrubar o obstáculo durantea fase deultrapassagem: aspecto que indica a existência de dificuldades na aval iação da profundidade elocalização precisa do obstáculo (apresentam variação de um sujeito para outro).

\section{Resultados e discussões}

\subsection{A nálise Qualitativa da tarefa}

Inicial mente foi realizada a análise qualitativa da locomoção de cada participante, com base nos 7 indicadores supracitados. O Quadro 1 apresenta os dados de cada sujeito facilitando a comparação das dificuldades específicas geradas por cada tipo depatologia. 
Quadro 1 - A val iação dos indicadores qual itativos para os sujeitos entre o préeo pós-teste.

\begin{tabular}{|c|c|c|c|c|c|c|c|c|c|c|c|c|}
\hline \multirow[b]{2}{*}{ Indicadores qualitativos } & \multirow{2}{*}{\multicolumn{2}{|c|}{1}} & \multicolumn{10}{|c|}{ Sujeitos } \\
\hline & & & Pré & 2 & & 3 & Pré & & $\begin{array}{r}5 \\
\text { Pré } \\
\end{array}$ & Pós & $\begin{array}{r}6 \\
\text { Pré }\end{array}$ & Pós \\
\hline $\begin{array}{l}1 \text { Manter o contato visual com o obstáculo } \\
\text { Durante todas as fases } \\
2 \text { Direção da caminhada - fase de aproximação }\end{array}$ & $\mathrm{S}$ & $S$ & $S$ & $S$ & $S$ & $S$ & $S$ & $\mathrm{~S}$ & $S$ & $\mathrm{~S}$ & $\mathrm{~S}$ & $S$ \\
\hline $\begin{array}{l}\text { Caminhada lateralizada } \\
3 \text { Equilíbrio durante a ultrapassagem }\end{array}$ & A & $\mathrm{N}$ & S & $\mathrm{N}$ & $\mathrm{N}$ & $\mathrm{N}$ & A & $\mathrm{N}$ & A & $\mathrm{N}$ & A & $\mathrm{N}$ \\
\hline $\begin{array}{c}\text { Utilização dos braços para equilibrar-se } \\
4 \text { Direção da passada - fase de ultrapassagem }\end{array}$ & A & $\mathrm{N}$ & $\mathrm{N}$ & $\mathrm{N}$ & A & $\mathrm{N}$ & A & $\mathrm{N}$ & $S$ & $\mathrm{~N}$ & $\mathrm{~N}$ & $\mathrm{~N}$ \\
\hline $\begin{array}{l}\text { Posição do pé lateralizada } \\
5 \text { Velocidade na execução da tarefa-teste }\end{array}$ & $\mathrm{S}$ & $\mathrm{N}$ & A & $\mathrm{N}$ & A & $\mathrm{N}$ & A & $\mathrm{N}$ & A & $\mathrm{N}$ & A & $\mathrm{N}$ \\
\hline $\begin{array}{l}\text { Executa a tarefa lentamente } \\
6 \text { Tocar ou derrubar o obstáculo }\end{array}$ & $\mathrm{S}$ & $\mathrm{N}$ & S & $\mathrm{N}$ & A & $\mathrm{N}$ & A & $\mathrm{N}$ & A & $\mathrm{N}$ & $\mathrm{S}$ & $\mathrm{N}$ \\
\hline Tocar ou derrubar o obstáculo & A & $\mathrm{N}$ & $\mathrm{N}$ & $\mathrm{N}$ & A & $\mathrm{N}$ & A & $\mathrm{N}$ & A & $\mathrm{N}$ & $\mathrm{N}$ & $\mathrm{N}$ \\
\hline
\end{tabular}

Todos os participantes mantiveram o contato visual tanto na aproximação quanto na ultrapassagem do obstáculo. Esteresultado, já esperado, reforça a característica da amostra que apresenta dificuldades na aquisição da informação visual, necessitando, portanto deum contínuo contato com o obstáculo. O uso contínuo da informação visual retirado texto se torna necessário quando a qualidade da informação visual é reduzida. Isto tem sido observado em estudos realizados com idosos saudáveis (CHA PMAN; HOLLANDS, 2006) e com idosos portadores de maculopatia (PATLA et al., 1995).

A mudança na direção da caminhada observada no pós-testeem quase todos os participantes sugere mel horas qual itativas na locomoção. A realização da passada de forma lateral izada durantea aproximação (observada muitas vezes no préteste) afeta a velocidade de progressão e também compromete a percepção sobre a posição e tamanho do obstáculo. Considerando que a baixa visão afeta diversos aspectos da percepção visual tais como a visão periférica, a acuidadevisual para longe, a noção de profundidade, a fixação entreoutros, pode-sesugerir quea passada lateralizada seria uma estratégia para compensar tais problemas.

Desta forma, os resultados observados no pós-teste sugerem que o programa de treinamento contribuiu para um mel hor controledas passadas fruto do enriquecimento da informação sensorial (resíduo visual e estímulo proprioceptivo).

Os indicadores relativos à fase de ultrapassagem do obstáculo mostram bastante alteração entreo prée o pós-teste. A perda do equilíbrio está relacionada com a execução da passada de forma lateralizada e também com a redução na velocidade de progressão. Tais alterações causaram mudanças significativas na ultrapassagem gerando inúmeros toques eal gumas vezes derrubando o obstáculo. 
Demaneira geral os indicadores qualitativos reforçam que o programa detreinamento contribui para um melhor planejamento eexecução da tarefa. Esta análiseéfortalecida através dos dados quantitativos referentes ao posicionamento do péna fase deaproximação ea el evação da perna durantea ultrapassagem.

\subsection{Análise dos resultados em relação às Variáveis biomecânicas}

O posicionamento do pé na fase de aproximação foi alterado. Houve uma redução na DPO em todas as condições de obstáculo (Tabela 1).

Tabela 1 - Diferença entre as médias obtidas no pós e no préteste de cada participantepara aDPO na fasedeaproximação.

\begin{tabular}{cccc}
\hline \multicolumn{4}{c}{$\begin{array}{c}\text { DISTÂNCIA PÉ-OBSTÁCULO (DPO) } \\
\text { Altura dos Obstáculos (cm) }\end{array}$} \\
\cline { 2 - 4 } Sujeitos & $\mathbf{5} \mathbf{~ c m}$ & $\mathbf{1 0} \mathbf{~ c m}$ & $\mathbf{1 5} \mathbf{~ c m}$ \\
\hline $\mathbf{1}$ & 8,22 & $-7,10$ & $-1,27$ \\
$\mathbf{2}$ & 3,07 & $-3,50$ & $-8,27$ \\
$\mathbf{3}$ & $-8,87$ & $-8,75$ & $-9,60$ \\
$\mathbf{4}$ & $-11,36$ & $-2,48$ & $-3,67$ \\
$\mathbf{5}$ & $-3,63$ & 6,43 & 3,04 \\
$\mathbf{6}$ & $-6,77$ & $-7,58$ & $-6,03$ \\
\hline
\end{tabular}

Deacordo com uma das hipóteses desteestudo, a diminuição (val ores negativos) na distância entre o péeo obstáculo no momento da aproximação para a ul trapassagem, indica que o sujeito tem maior segurança na real ização da tarefa. Posicionar o pé de apoio mais próximo do obstáculo favorece a fase de vôo da perna que ultrapassa primeiro o obstáculo.

Segundo estudo realizado por Patla et al. (1991), a real ização de uma ultrapassagem segura podeser garantida por meio de duas estratégias: via el evação da perna através de uma mai or flexão das articulações do quadril e do joelho ou via el evação de todo o corpo através da elevação do centro de gravidade gerado por um maior impulso vertical na perna de apoio. A primeira estratégia produz efeitos locais, enquanto que a segunda afeta o equilíbrio corporal total.

As análises qualitativa e biomecânica sugerem que as crianças com baixa visão realizam uma combinação das estratégias propostas por Patla et al (1991). O posicionamento do pémais distante do obstáculo forçou os participantes a real izarem tanto uma mai or el evação da perna quanto uma elevação do centro degravidade. Embora não tenha sido coletado dados específicos sobre o cento de gravidade, os indicadores qualitativos mostram que muitos participantes utilizaram os braços para se manterem em equilíbrio durante a ultrapassagem. Isto mostra que a aproximação indevida gerou mudanças globais na locomoção. 
Pode-se observar também uma diminuição na EMJ (Tabela 2) no momento da ultrapassagem dos obstáculos, quando comparados os dados do pré e o do pós-teste para cada sujeito.

Tabela 2 - Diferença entre as médias obtidas no pós e no préteste de cada participante para a EMJ no momento da ul trapassagem dos obstáculos.

\begin{tabular}{cccc}
\hline & \multicolumn{3}{c}{$\begin{array}{c}\text { ELEVAÇÃo MÁXIMA DO JOELHO (EMJ) } \\
\text { Altura dos }\end{array}$} \\
\cline { 2 - 4 } Sujeitostáculos (cm) \\
\cline { 2 - 4 } & $\mathbf{5 c m}$ & $\mathbf{1 0} \mathbf{c m}$ & $\mathbf{1 5} \mathbf{~ c m}$ \\
\hline $\mathbf{1}$ & $-1,47$ & $-11,7$ & $-10,13$ \\
$\mathbf{2}$ & $-6,17$ & $-14,53$ & -11.0 \\
$\mathbf{3}$ & $-15,87$ & $-17,4$ & $-11,63$ \\
$\mathbf{4}$ & $-14,17$ & $-17,73$ & $-9,6$ \\
$\mathbf{5}$ & $-12,1$ & $-11,18$ & $-12,1$ \\
$\mathbf{6}$ & $-7,87$ & $-4,58$ & $-4,83$ \\
\hline
\end{tabular}

A diminuição (val ores negativos) na EMJ durantea ultrapassagem do obstáculo, indica que os participantes adequaram o movimento da perna deacordo com a al tura do obstáculo reduzindo a el evação desnecessária que comprometeo equilíbrio e a continuidade da locomoção.

A seguir apresentamos a média e o desvio padrão das variáveis considerando todos os participantes. O objetivo desta análise é identificar uma tendência geral de mudanças nestas variáveis (Tabela 3).

Tabela 3 - Média e desvio padrão da DPO, EMJ e Toe Clearance de todos os participantes nas três alturas de obstáculos.

\begin{tabular}{|c|c|c|c|c|c|c|}
\hline \multirow[b]{2}{*}{$\begin{array}{l}\text { Altura do } \\
\text { Obstáculo }\end{array}$} & \multicolumn{2}{|c|}{$\mathrm{DPO}(\mathrm{cm})$} & \multicolumn{2}{|c|}{ EMJ (cm) } & \multicolumn{2}{|c|}{ Toe Clearance (cm) } \\
\hline & Pré-teste & Pós-teste & Pré-teste & Pós-teste & Pré-teste & Pós-teste \\
\hline & $\mathrm{M} \pm \mathrm{STD}$ & $\mathrm{M} \pm \mathrm{STD}$ & $\mathrm{M} \pm \mathrm{STD}$ & $\mathrm{M} \pm \mathrm{STD}$ & $\mathrm{M} \pm \mathrm{STD}$ & $\mathrm{M} \pm \mathrm{STD}$ \\
\hline $5 \mathrm{~cm}$ & $16,88 \pm 4,02$ & $13,82 \pm 3,42$ & $59,23 \pm 3,80$ & $49,60 \pm 3,40$ & $7,1 \pm 2,36$ & $9,3 \pm 2,3$ \\
\hline $10 \mathrm{~cm}$ & $18,97 \pm 6,88$ & $15,47 \pm 3,77$ & $63,13 \pm 3,73$ & $51,13 \pm 4,73$ & $6,88 \pm 2,63$ & $8,08 \pm 2,96$ \\
\hline $15 \mathrm{~cm}$ & $17,62 \pm 5,38$ & $13,30 \pm 4,27$ & $65,53 \pm 5,35$ & $55,63 \pm 2,78$ & $5,83 \pm 1,95$ & $7,26 \pm 3,43$ \\
\hline
\end{tabular}

Os dados pré e pós mostram que em média a DPO reduziu diminuiu em todas as condições experimentais. $\mathrm{Na}$ condição com obstáculo de $5 \mathrm{~cm}$ a redução foi de $18,13 \%$ (de 16,88 cm para 13,82 cm), na condição com obstáculo de $10 \mathrm{~cm}$ a redução foi um pouco maior $18,45 \%$ (de $18,97 \mathrm{~cm}$ para $15,47 \mathrm{~cm}$ ), ecom obstáculo 
de 15 cm a redução foi ai nda maior reduzindo aproximadamente24,52\% (de 17,62 cmpara $13,3 \mathrm{~cm})$.

A análise da EMJ também mostrou uma redução nos valores prée pós treinamento. N o obstácul o com $5 \mathrm{~cm}$ a redução foi de $16,26 \%$ (59,23 cm para 49,60 cm); no obstáculo de $10 \mathrm{~cm}$ a redução foi de $20,43 \%$ (63,13 cm para 50,23 cm); eno obstáculo de $15 \mathrm{~cm}$ a redução foi de $15 \%$ (65,53 cm para 55,63cm). Pode-seobservar também que houveum aumento na elevação máxima da perna, na medida em que a altura do obstáculo aumentou.

Isto sugerequeos participantes, embora com baixa visão, foram capazes de perceber as diferenças entre as al turas dos obstáculos. Tem sido observado na literatura queo ajuste na el evação máxima do joel ho ocorre como uma estratégia para garantir uma margem de segurança durante a ultrapassagem do obstáculo (MOHAGHEGHI; MORAES; PATLA, 2003).

A margem de segurança (Toe Clearance) mostrou que apesar de os participantes reduzirem a el evação máxima da perna eles mantiveram uma boa distância em relação ao topo do obstáculo.

Os dados apresentados na Tabela 3 mostram que após o programa houve uma maior el evação do pé no momento da ultrapassagem do obstáculo. Esta al teração correspondeu a 30,98\% para o obstácul o com 5 cm, 17,44\% para o obstáculo com $10 \mathrm{~cm}$, e 5,21\% para o obstáculo com $15 \mathrm{~cm}$. Estes dados sugerem que os participantes realizaram uma maior dorsiflexão no momento da ultrapassagem do obstáculo, o que evidencia um controle mais preciso da articulação do tornozelo para evitar o toque do pé no obstáculo.

A redução devalores, em relação à EMJ, revela que o treinamento foi importante para a ultrapassagem do obstáculo, ou seja, contribuiu principalmente para o controle contínuo da el evação da pena, tornando a execução mais precisa.

Estudo realizado por Patla (1997), mostrou quea visão intermitenteé adequada para executar a locomoção em vários terrenos. Porém, os dados apresentados nesteestudo com crianças com baixa visão, demonstram a necessidade da utilização da informação visual deforma contínua, ou seja, houvea necessidade de manter o contato visual com o obstáculo durante todo o percurso.

Tal necessidadeé semelhante ao observado por Chapman eHollands (2006) em estudo real izado com idosos sobre a locomoção sobre alvos desenhados no solo. Eles observaram que devido ao declínio do controle visuo-motor causado pelo processo de envelhecimento os idosos necessitaram da informação visual durantevários momentos dentro do ciclo da passada.

Os resultados mostraram quea remoção da informação visual durante a fase de vôo gerou um erro no posicionamento do pé de $23 \%$. O erro foi ainda maior (42\%) quando a informação visual foi removida duranteafasedeapoio. Os resultados deste estudo reforçam a noção de que o uso da informação visual de 
forma contínua (On-line) setorna importante para os idosos eprincipalmente para aqueles que possuem capacidade visual reduzida.

A análise da variabilidade da EMJ e da DPO mostrou também uma grande redução entre o prée o pós-teste. $\mathrm{NaEMJ}$ a variabilidade foi reduzida em 10,53\% com obstáculo de 5 cm; em 9,02\% com o obstáculo de 10 cm; e em 48,4\% para o obstáculo de $15 \mathrm{~cm}$. A variabilidade da DPO diminuiu em todas as condições (5cm: 14,93\%; 10 cm: 45,2\%; e 15 cm: 20,63\%).

Na variável T oe Clearance a variabilidade permaneceu a mesma nas condições com obstáculo de 5 e $10 \mathrm{~cm}$ eaumentou na condição com obstáculo de $15 \mathrm{~cm}$. A redução da variabilidade observada neste estudo representa um mel hor controletanto da aproximação quanto da ultrapassagem do obstáculo.

A redução da variabilidade tanto da DPO quanto da EMJ é um bom indicador sobre a melhoria do controle da locomoção sobre obstáculos. Estudo recenterealizado por PatlaeGreig (2006) mostrou quea manipulação da informação visual duranteas últimas três passadas antes da ultrapassagem do obstáculo afeta significativamente o sucesso na tarefa. Os autores observaram uma redução da variabilidade no posicionamento do pé na medida em que este se aproximou do obstáculo.

Os dados sobre a elevação da perna demonstraram que após o treinamento, houve um melhor controle da perna durante a ultrapassagem dos obstáculos. Portanto, os participantes utilizaram al ém do resíduo visual, outras fontes de informações sensoriais, como a propriocepção, provavelmente adquiridas duranteo treinamento, para regular o movimento do próprio corpo, evitando assim, um gasto desnecessário de energia.

Segundo Patla (1998), as informações externas (presença de obstáculos e características do terreno) sobre o ambiente são utilizadas de forma antecipada (F eed-forward) para o planejamento da ul trapassagem de obstáculos. Os resultados desteestudo mostram queas crianças com baixa visão real izaram um planejamento antecipado, entretanto, necessitaram de um controle contínuo tanto durante a aproximação quanto durantea execução para ajustar os movimentos corporais em relação à demanda da tarefa. Isto foi observado pela contínua manutenção do contato visual com o obstáculo, durante a fase de aproximação etambém durante a fase deultrapassagem.

\section{Conclusões}

Os resultados indicam que, durantea ultrapassagem de um obstáculo, crianças com baixa visão necessitam real izar um ajustamento contínuo do controle motor, visto que as informações visuais adquiridas no momento da aproximação do obstáculo não bastam para um planejamento antecipado da tarefa motora. 
Mesmo com pouco tempo de treinamento, o programa composto de atividades simples, geralmente presentes num ambiente rico em vivências corporais, mostrou-se eficientee contribuiu para a realização da ultrapassagem do obstáculo, conforme observado na redução da el evação do joelho, e, conseqüentemente, para conscientização corporal das crianças.

Sendo assim, o trabalho educacional com crianças portadoras debaixa visão deveressal tar a necessidade do enriquecimento proprioceptivo, para ampliar as fontes de informações sensoriais e propiciar mais segurança e independência na locomoção, principal mente nas aulas de Educação Física.

As crianças com baixa visão devem, portanto, ser submetidas a um programa especial de estimulação, a ser desenvolvido tanto na escola como no próprio ambiente familiar, queauxiliena ampliação do repertório de experiências corporais. Cumpre destacar quea característica central dessas experiências, segundo a proposta educativa da psi comotricidade, deve estar pautada na diversificação dos estímulos, deforma a apresentar para criança desafios cada vez mais complexos, que ela deve ser capaz de resolver de acordo com as suas habilidades, colocando em jogo as funções de ajustamento e organização perceptiva sugeridas por Le Boulch (1988) que correspondem aos dois processo complementares e concomitantes sugeridos por Piaget: assimilação eacomodação.

As contribuições advindas dessetipo de programa de estimulação não se restringem ao controle visual da locomoção, pois existe uma interação muito próxima entrevisão edesenvolvimento, deforma quea aprendizagem psicomotora amplia consideravelmente as possibilidades da criança descobrir e, progressivamente, dominar um conjunto de habilidades relacionadas com seu próprio corpo, com os objetos e suas diferentes características, com as dimensões espaciais e temporais, como também, com a comunicação e socialização com o outro, seja ele adulto ou criança.

Estudos posteriores devem investir na discussão dos estímulos que possuem um papel chave para melhor adequação dos programas de educação precoce e de reabilitação para as crianças com baixa visão. A escola, ao ensinar a criança a se movimentar ea usar o resíduo visual, contribui para a formação de um sentimento deautoconfiança e para a mel horia de suas habilidades gerais, dando suporte para que adote uma postura comprometida com uma ação exploratória e, na medida do possível, independente que, a longo prazo, representa uma verdadeira conquista da cidadania e da inserção social.

Estudos sobre este assunto são de fundamental importância para um melhor entendimento do controle motor em portadores de deficiência visual, pois a locomoção segura eindependenteéum avanço importantena qualidade devida dessas pessoas. 


\section{Referências}

AMARAL, A.C.T.; TABAQUIM, M.L.M.;LAMONICA, D.A.C. Avaliação das habilidades cognitivas, da comunicação e neuromotoras de crianças com risco de alterações do desenvolvimento. Revista Brasileira Educação Especial, Marília, v.11, n.2, p.185-200, 2005.

AMERICAN PSYCHIATRIC ASSOCIATION (APA). M anual de diagnóstico e estatística das perturbações mentais dsm IV . 4. ed. A merican Psychiatric Publishing, INC. (APPI). 1996.

CAVALCANTE, A.M.M. Educação visual: atuação na pré-escola. R evista Benjamin Constant, Rio de Janeiro, v.1, p. 11-26, 1995.

CHAPMAN, G.J.; HOLLANDS, M.A. Age-related differences in stepping performance during step cycle-related removal of vision. Experimental Brain Research, Heidelberg, v. 174, p.613-621, 2006.

CHOU, L.S.; DRA GANICH, L.F. Placing thetrailing foot closer to an obstaclereducesflexion of hip, knee, and ankle to increase the risk of tripping. Journal of Biomechanics, Elmsford, $v$. 31, p. 685-691, 1998 a.

. Increasing obstacle height and decreasing toe-obstacle distance affect the joint moments of the stance limb differently when stepping over an obstacle. Gait and Posture, Oxford, v. 8, p. 186-204, 1998 b.

HUEARA, L.; SOUZA, C.M.L.; BATISTA, C.G. O faz-de-conta em crianças com deficiência visual: identificando habilidades. Revista Brasileira E ducação Especial, Marília, v.12, n.3, p.351368, 2006.

LE BOULCH, J. Educação psicomotora: psicocinética na idade escolar. 2. ed. Porto Alegre: Artes Médicas, 1988.

MINISTÉRIO DA EDUCAÇÃO/ SECRETARIA DE EDUCAÇÃO ESPECIAL. Programa de Capacitação de Recursos Humanos do Ensino Fundamental: deficiência visual. v. 1, Fascículos I - II - III / Marilda Moraes Garcia Bruno, Maria Glória Batista da Mota, colaboração: Instituto Benjamin Constant. Série A tual idades Pedagógicas; 6. Brasilia, 2001.

MOHAGUEGHI, A.A.; MORAES, R.; PATLA, A.E. The effects of distant and on-line visual information on the control of approach phase and step over an obstacle during locomotion. Experimental Brain Research, Heidelberg, v. 155, n.4, p. 459-468, 2003.

PATLA, A.E. Understanding the roles of vision in the control of human locomotion. Gait and Posture, Oxford, v. 5, p. 54-69, 1997.

. How is human gait controlled by vision? Ecological Psychology, New Jersey, v. 10, n.3-4, p. 287-302, 1998.

PATLA, A.E.; GREIG, M. Any way you look at it, successful obstacle negotiation needs visually guided on-line foot placement regulation during the approach phase. N euroscience Letters, San Diego, v. 397, p.110-114, 2006.

PATLA, A.E. et al. Visual control of locomotion: strategies for changing direction and for going over obstacles. Journal of Experimental Psychology: Human Perception and Performance, Washington, v. 17, n.3, p.603-634, 1991.

PATLA, A.E. et al. Effects of age related maculopathy on strategies for going over obstacles of different heights and contrast. Gait and Posture, Oxford, v. 3, n. 2, p.106, 1995. 
SANTOS, L. C.; PASSOS; J. E. O. S.; REZENDE, A. L. G.

PATLA, A.E.; RIETDYJK, S. Visual control of limb trajectory over obstacles during locomotion: effect of obstacle height and width. Gait and Posture, Oxford, v. 1, p. 45-60, 1993.

SOCIEDADE BRASILEIRA DE VISÃO SUBNORMAL. Base de dados. Disponível em: বtttp:/ / www.cbo.com.br/ subnorma/ conceito.htm>. A cesso em: 19 de jul. 2007.

SOUZA , A. Propriocepção. Rio de Janeiro: Editora Médica e Científica Ltda, 2004.

Recebido em 04/ 05/ 2007

Reformulado em 23/ 07/ 2007

A provado em 30/ 09/ 2007 